\title{
ANALYSIS RESULTS FOR DYNAMIC CONTACT PROBLEM WITH FRICTION IN THERMO-VISCOELASTICITY
}

\author{
MUSTAPHA BOUALLALA AND EL HASSAN ESSOUFI
}

\begin{abstract}
We present a mathematical model which describes the dynamic frictional contact between a thermo-viscoelastc body and a conductive foundation. The contact is modeled using the normal compliance condition, the quasistatic version of Coulomb's law of fry friction. We derive the weak formulation and we prove the existence and uniqueness result. The proofs are based on the theory of first-order and second-order evolution inequalities and Banach fixed point theorem. We introduce a new problem on perturbation of the contact boundary condition and we establish its continuous dependence result.

Представлено математичну модель, що описує динамічний контакт тертя між термов'язкопружним тілом і основою-провідником. Контакт моделюється з використанням умови нормальної еластичності, квазістатичної версії закону фрикційного тертя Кулона. Пропонується означення слабкого розв'язку, доведена теорема існування та єдиності. Доведення базуються на теорії еволюційних нерівностей першого та другого порядків і теоремі Банаха про нерухому точку. Ставиться нова задача про збурення контактної граничної умови; отримана теорема про неперервну залежність.
\end{abstract}

\section{INTRODUCTION}

The contact of deformable elastic bodies, with or without friction, is the relationship between the normal forces (contact pressure) and the relative movement between the body and the foundation. Contact problems are present in many industrial processes and daily life.

Dynamic frictional contact problems between a viscoelastic body and an obstacle with thermal effect was made in [6, 3, 7] and more recently in [1, 21]. The result in 14, 15, 16, 17, we can find several classes of dynamic frictional contact problems by hemivariational inequalities with or without thermal effects. For more information of the quasistatic and dynamic problem without thermal effects refer to [18, 9, 10]. A number of papers investigating quasistatic thermo-viscoelastic contact problems with friction can be found in 2, 13, 19.

Essoufi et.al [12, 11] study two dynamic frictional contact models between a piezoelectric and electro-viscoelastic body and a rigid, non conductive obstacle, using Tresca and Coulomb model for the contact. The authors in 44 introduced an evolution quasistatic contact problem of a viscoelastic body with a foundation, they modeled the contact with normal damped response and a local friction law. They established the existence of a unique solution and the continuous dependence of the solution on the contact boundary conditions.

In this paper, we study the process of dynamical frictional contact between a thermoviscoelastic body and a rigid foundation which, is thermally conducting. We assume that the process is dynamic and the contact is modeled with the normal compliance,

2020 Mathematics Subject Classification. 35J85. 47H10. 47H05. 74M10. 74M15. 35D30.

Keywords. Thermo-viscoelastic, normal compliance, Coulomb's friction, weak solvability, monotone operator, fixed point. 
the quasistatic version of Coulomb's law of dry friction, a regularizing condition of thermal contact. The weak variational formulation which consists of a system coupling a variational inequality for displacement field and a parabolic variational equality for the temperature. We establish the existence of unique solution using Banach fixed theorems and we prove the dependence of the solution on the contact boundary conditions and its convergence result.

The rest of the paper is structured as follows. In section 2, we describe the dynamic model of the frictional thermo-viscoelastic contact problem. In section 3 , we list the assumptions of the problem data, we derive its variational formulation. In section 4 , we state and prove our existence and uniqueness result. In the last section we study a contact problem for thermo-electro-viscoelastic with a small perturbation of the contact condition and we prove a convergence result.

\section{Problem statement}

We consider a body made of thermo-viscoelastic material which occupies in the reference configuration the domain $\Omega \subset \mathbb{R}^{d}, d=1,2,3$, which will be supposed bounded with a smooth boundary $\partial \Omega=\Gamma$ that is divided into three disjoint measurable parts $\Gamma_{N}, \Gamma_{D}$ and $\Gamma_{C}$ such that meas $\left(\Gamma_{D}\right)>0$. Let $T>0$ and $[0, T]$ be the time interval of and $\rho: \Omega \mapsto \mathbb{R}^{+}$mass density of the body. The body is clamped on $\Gamma_{D} \times(0, T)$ and so the displacement field vanish there; a volume force of density $f_{0}$ acts in $\Omega \times(0, T)$ and a volume thermal of density $q_{0}$. Surface traction of density $f_{1}$ act on $\Gamma_{N} \times(0, T)$. In $\Gamma_{C} \times(0, T)$ the body can arrive in frictional contact with the so called foundation which is thermally conductive. The variation of temperature is assumed to be zero on $\Gamma_{D} \cup \Gamma_{N} \times(0, T)$. We assume that the thermal potentials is maintained fix on $\theta_{F}$. The normalized gap between $\Gamma_{C} \times(0, T)$ and the rigid foundation is denoted by $g$.

The space of second order symmetric tensors in the space $\mathbb{R}^{d}$ is denoted by $\mathbb{S}^{d}$ while "." and $\|$.$\| will represent the inner product and the Euclidean norm on \mathbb{R}^{d}$ and $\mathbb{S}^{d}$, that is $\forall u, v \in \mathbb{R}^{d}, \forall \sigma, \tau \in \mathbb{S}^{d}$

$$
u . v=u_{i} \cdot v_{i}, \quad\|v\|=(v, v)^{\frac{1}{2}} \quad \text { and } \quad \sigma \cdot \tau=\sigma_{i j} \cdot \tau_{i j}, \quad\|\tau\|=(\tau, \tau)^{\frac{1}{2}} .
$$

We use the notation $u_{\nu}$ and $u_{\tau}$ for the normal and tangential displacement that is $u_{\nu}=u . \nu$ and $u_{\tau}=u-u_{\nu} \nu$. We also denote by $\sigma_{\tau}$ the normal and tangential stress give by $\sigma_{\nu}=\sigma \nu . \nu, \sigma_{\tau}=\sigma \nu-\sigma_{\nu} \nu$.

The classical formulation of the thermo-viscoelastic contact problem is as follows:

- Problem $(P)$ : Find a displacement field $u: \Omega \times] 0, T\left[\longrightarrow \mathbb{R}^{d}\right.$ and a temperature field $\theta: \Omega \times] 0, T[\longrightarrow \mathbb{R}$ such that

$$
\begin{aligned}
& \sigma(t)=\mathcal{A} \varepsilon(u(t))+\mathcal{B} \varepsilon(\dot{u}(t))-\theta(t) \mathcal{M} \quad \text { in } \quad \Omega \times(0, T), \\
& q(t)=-\mathcal{K} \nabla \theta(t) \quad \text { in } \quad \Omega \times(0, T), \\
& \rho \ddot{u}(t)=\operatorname{Div} \sigma(t)+f_{0}(t) \quad \text { in } \quad \Omega \times(0, T), \\
& \dot{\theta}(t)+\operatorname{div} q(t)-\mathcal{R} \varepsilon(u(t))=q_{0}(t) \quad \text { in } \quad \Omega \times(0, T), \\
& u=0 \quad \text { on } \quad \Gamma_{D} \times(0, T), \\
& \sigma(t) \nu=f_{1}(t) \quad \text { on } \quad \Gamma_{N} \times(0, T) \text {, } \\
& \theta=0 \quad \text { on }\left(\Gamma_{N} \cup \Gamma_{D}\right) \times(0, T), \\
& u(0, x)=u_{0}, \dot{u}(0, x)=\dot{u}_{0}, \theta(0, x)=\theta_{0} \quad \text { in } \quad \Omega, \\
& -\sigma_{\nu}(u(t)-g)=p_{\nu}\left(u_{\nu}(t)-g\right), \quad \text { on } \quad \Gamma_{C} \times(0, T),
\end{aligned}
$$




$$
\begin{aligned}
& \left.\begin{array}{l}
\left\|\sigma_{\tau}(t)\right\| \leq p_{\tau}\left(u_{\nu}(t)-g\right), \\
\dot{u}_{\tau}(t) \neq 0 \Rightarrow \sigma_{\tau}(t)=-p_{\tau}\left(u_{\nu}(t)-g\right) \frac{\dot{u}_{\tau}(t)}{\left\|\dot{u}_{\tau}(t)\right\|},
\end{array}\right\} \text { on } \Gamma_{C} \times(0, T) \\
& \frac{\partial q(t)}{\partial \nu}=k_{c}\left(u_{\nu}(t)-g\right) \phi_{L}\left(\theta(t)-\theta_{F}\right) \quad \text { on } \quad \Gamma_{C} \times(0, T),
\end{aligned}
$$

where (2.1) and (2.2) are the thermo-viscoelastic constitutive law of the material, $\sigma=$ $\left(\sigma_{i j}\right): \Omega \times(0, T) \rightarrow \mathbb{S}^{d}$ the stress tensor, $q=\left(q_{i}\right): \Omega \times(0, T) \rightarrow \mathbb{R}^{d}$ the heat flux vector, $\varepsilon(u)=\left(\varepsilon_{i j}(u)\right)=\frac{1}{2}\left(u_{i . j}+u_{j . i}\right), \mathcal{A}=\left(\mathcal{A}_{i j k l}\right), \mathcal{B}=\left(\mathcal{B}_{i j k l}\right), \mathcal{M}=\left(\mathcal{M}_{i j}\right)$ and $\mathcal{K}=\left(\mathcal{K}_{i j}\right)$ are respectively the linearized strain tensor, elastic tensor, (fourth-order) viscosity tensor, thermal expansion tensor and thermal conductivity tensor.

Equation (2.3) is the equation of motion. Equation (2.4) is the Fourier law of heat conduction where the function $\mathcal{R}=\left(\mathcal{R}_{i j}\right)$ describe the influence of the displacement field. Here and below Div $\sigma=\sigma_{i j . j}$ and div $q=\left(q_{i, i}\right)$ denote the divergence operator for tensor and vector valued functions. Conditions (2.5)-(2.7) are the displacement and thermal boundary condition. The initials conditions are represented in the equation (2.8). Relation (2.9) represents the normal compliance contact condition, where $p_{\nu}$ is a prescribed function. When it is positive, $u_{\nu}-g$ represents the penetration of the surface as parities into those of the foundation. The Coulomb's law of friction is considered in relation (2.10) where $p_{\tau}$ is a prescribed non-negative function, the so-called friction bound. Finally, the relation (2.11) represents a regularized thermal contact condition where $\frac{\partial q}{\partial \nu}$ is the normal derivative of $q$ such that:

$$
\phi_{L}(s)=\left\{\begin{array} { c l c } 
{ - L } & { \text { if } } & { s < - L , } \\
{ s } & { \text { if } } & { - L \leq s \leq L , } \\
{ L } & { \text { if } } & { s > L , }
\end{array} \quad \left\{\begin{array}{ccc}
k_{c}(r)=0 & \text { if } & r<0 \\
k_{c}(r)>0 & \text { if } & r \geq 0
\end{array}\right.\right.
$$

where $L$ is a large positive constant, see [8].

\section{VARIATIONAL FORMULATION}

In order to obtain the variational formulation of Problem $(P)$, let us denote by

$$
\begin{array}{cl}
H=\left[L^{2}(\Omega)\right]^{d}=\left\{u=\left(u_{i}\right): u_{i} \in L^{2}(\Omega)\right\}, & \mathcal{H}=\left\{\sigma=\left(\sigma_{i j}\right): \sigma_{i j}=\sigma_{j i} \in L^{2}(\Omega)\right\}, \\
H_{1}=\left\{u=\left(u_{i}\right): \varepsilon(u) \in \mathcal{H}\right\}=\left[H^{1}(\Omega)\right]^{d}, & \mathcal{H}_{1}=\{\sigma \in \mathcal{H}: \text { Div } \sigma \in H\} .
\end{array}
$$

These are real Hilbert spaces endowed with the inner products

$$
\begin{array}{cl}
(u, v)_{H}=\int_{\Omega} u_{i} v_{i} d x, \forall u, v \in H, & (\sigma, \tau)_{\mathcal{H}}=\int_{\Omega} \sigma_{i j} \tau_{i j} d x, \forall \sigma, \tau \in \mathcal{H}, \\
(u, v)_{H_{1}}=(u, v)_{H}+(\varepsilon(u), \varepsilon(v))_{\mathcal{H}}, & (\sigma, \tau)_{\mathcal{H}_{1}}=(\sigma, \tau)_{\mathcal{H}}+(\operatorname{Div} \sigma, \text { Div } \tau)_{H},
\end{array}
$$

and the associated norms, $\|\cdot\|_{H},\|\cdot\|_{H_{1}},\|\cdot\|_{\mathcal{H}}$, and $\|\cdot\|_{\mathcal{H}_{1}}$.

Let $X$ be a Banach space. For every $1 \leq p \leq \infty$, we will use the spaces $L^{p}(0, T ; X)$, $C(0, T ; X)$ and $W^{k, p}(0, T ; X)$ with their standard norm.

Moreover, keeping in mind (2.5) and (2.7), we introduce the following space

$$
\begin{gathered}
V=\left\{v \in H_{1}(\Omega): v=0 \text { on } \Gamma_{D}\right\}, V_{a d}=\left\{v \in V: u_{\nu} \leq g \text { on } \Gamma_{C}\right\}, \\
Q=\left\{\eta \in H_{1}(\Omega): \eta=0 \text { on } \Gamma_{D} \cup \Gamma_{N}\right\} .
\end{gathered}
$$

endowed with the inner products and norms given by

$$
\begin{aligned}
(u, v)_{V}=(\varepsilon(u), \varepsilon(v))_{\mathcal{H}}, & \|v\|_{V}=(v, v)_{V}^{\frac{1}{2}}, \\
(\theta, \eta)_{Q}=(\nabla \theta, \nabla \eta)_{H}, & \|\eta\|_{Q}=(\eta, \eta)_{Q}^{\frac{1}{2}} .
\end{aligned}
$$


Since meas $\left(\Gamma_{D}\right)>0$, the following Korn's inequality holds

$$
\|\varepsilon(v)\|_{\mathcal{H}} \geq C_{K}\|v\|_{H_{1}}, \forall v \in V
$$

where $C_{K}>0$ is a constant which depends only on $\Omega$ and $\Gamma_{D}$.

The following Frierichs-Poincaré inequality holds on $Q$ at the bottom

$$
\|\nabla \eta\|_{H} \geq C_{F}\|\eta\|_{Q}, \forall \eta \in Q .
$$

Using Sobolev trace theorem, we have constants $C_{S_{1}}$ and $C_{S_{2}}$ depends only on $\Omega, \Gamma_{D}$ and $\Gamma_{C}$ such that

$$
\|v\|_{\left[L^{2}\left(\Gamma_{C}\right)\right]^{d}} \leq C_{S_{1}}\|v\|_{V}, \forall v \in V \text { and }\|\eta\|_{\left[L^{2}\left(\Gamma_{C}\right)\right]} \leq C_{S_{2}}\|\eta\|_{Q}, \forall \eta \in Q .
$$

Next, we define the elements $f(t) \in V$ and $q(t) \in Q$ respectively by

$$
\begin{aligned}
(f(t), v)_{V} & :=\int_{\Omega} f_{0}(t) \cdot v d x+\int_{\Gamma_{N}} f_{1}(t) \cdot v d a, \forall v \in V . \\
\left(q_{t h}(t), \eta\right)_{Q} & :=\int_{\Omega} q_{0}(t) \eta d x, \forall \eta \in Q .
\end{aligned}
$$

We consider $j: V \times V \rightarrow \mathbb{R}, \chi: V \times Q \times Q \rightarrow \mathbb{R}$ as follows:

$$
\begin{aligned}
j(u(t), v) & :=\int_{\Gamma_{C}} p_{\nu}\left(u_{\nu}(t)-g\right) v_{\nu} d a+\int_{\Gamma_{C}} p_{\tau}\left(u_{\tau}(t)-g\right)\left\|v_{\tau}\right\| d a, \\
\chi(u(t), \theta(t), \eta) & :=\int_{\Gamma_{C}} k_{c}\left(u_{\nu}(t)-g\right) \phi_{L}\left(\theta(t)-\theta_{F}\right) \eta d a,
\end{aligned}
$$

and the following operators

$$
\begin{aligned}
& a: V \times V \rightarrow \mathbb{R}, \quad a(u, v) \quad:=(\mathcal{A} \varepsilon(u), \varepsilon(v))_{\mathcal{H}}, \\
& b: V \times V \rightarrow \mathbb{R}, \quad b(u, v) \quad:=(\mathcal{B} \varepsilon(u), \varepsilon(v))_{\mathcal{H}}, \\
& d: Q \times Q \rightarrow \mathbb{R}, \quad d(\theta, \eta) \quad:=(\mathcal{K} \nabla \theta, \nabla \eta)_{H}, \\
& m: Q \times V \rightarrow \mathbb{R}, \quad m(\theta, v):=(\mathcal{M} \theta, \varepsilon(v))_{\mathcal{H}}, \\
& e: V \times Q \rightarrow \mathbb{R}, \quad e(u, \eta) \quad:=(\mathcal{R} \varepsilon(v), \eta)_{L^{2}(\Omega)} .
\end{aligned}
$$

Now, in order to study the problem (2.1)-(2.11), we need the following hypotheses on the data

(H1) The operators $a, b, d, m$ and $e$ satisfy the usual property of symmetry

$$
\begin{gathered}
\mathcal{A}_{i j k l}=\mathcal{A}_{j i k l}=\mathcal{A}_{l k i j} \in L^{\infty}(\Omega), \quad \mathcal{B}_{i j k l}=\mathcal{B}_{j i k l}=\mathcal{B}_{l k i j} \in L^{\infty}(\Omega), \\
\mathcal{K}_{i j}=\mathcal{K}_{j i} \in L^{\infty}(\Omega), \quad \mathcal{M}_{i j}=\mathcal{M}_{j i} \in L^{\infty}(\Omega), \quad \mathcal{R}_{i j}=\mathcal{R}_{j i} \in L^{\infty}(\Omega) .
\end{gathered}
$$

(H2) There exist positive constants $m_{a}, m_{b}$ and $m_{d}$ such that

$$
a(v, v) \geq m_{a}\|v\|_{V}^{2}, \quad b(v, v) \geq m_{b}\|v\|_{V}^{2}, \quad d(\eta, \eta) \geq m_{d}\|\eta\|_{Q}^{2} .
$$

(H3) The operators $a, b, d, m$ and $e$ satisfy the usual property of boundedness

$$
\begin{gathered}
|a(u, v)| \leq M_{a}\|u\|_{V}\|u\|_{V}, \quad|b(u, v)| \leq M_{b}\|u\|_{V}\|v\|_{V}, \\
|d(\theta, \eta)| \leq M_{d}\|\theta\|_{Q}\|\eta\|_{Q},|m(\theta, v)| \leq M_{m}\|\theta\|_{Q}\|v\|_{V},|e(u, \eta)| \leq M_{e}\|u\|_{V}\left\|_{\eta}\right\|_{Q},
\end{gathered}
$$

$(\mathrm{H} 4)$ i) The forces, the traction and the thermal flux satisfy

$f_{0} \in W^{1,1}\left(0, T ; L^{2}(\Omega)^{d}\right), \quad f_{1} \in W^{1,1}\left(0, T ; L^{2}\left(\Gamma_{N}\right)^{d}\right)$ and $q_{0} \in L^{2}\left(0, T ; L^{2}(\Omega)\right)$.

ii) The gap function and the thermal potential satisfy

$$
g \geq 0, g \in L^{\infty}\left(\Gamma_{C}\right) \text {, and } \theta_{F} \in L^{2}\left(0, T ; L^{2}\left(\Gamma_{C}\right)\right) .
$$

iii) The mass density $\rho$ satisfies $\rho \in L^{\infty}(\Omega)$, and there exists $\rho^{*}>0$ such that $\rho(x) \geq \rho^{*}$ a.e. $x \in \mathbb{R}$.

iv) The functional $j$ is proper, convex and lower semi-continuous on $V$. 
(H5) The coefficient of heat exchange $k_{c}: \Gamma_{C} \times \mathbb{R} \rightarrow \mathbb{R}^{+}$satisfy

i) There exists $M_{k_{c}}>0$ such that $\left|k_{c}(x, u)\right|<M_{k_{c}}$ for all $u \in \mathbb{R}, x \in \Gamma_{C}$ $x \mapsto k_{c}(x, u)$ is measurable on $\Gamma_{C}$ for all $x \in \mathbb{R}, k_{c}(x, u)=0$ for all $x \in \Gamma_{C}$ and $u \leq 0$.

ii) There exists $L_{k_{c}}>0$ such that $\left|k_{c}\left(x, u_{1}\right)-k_{c}\left(x, u_{2}\right)\right| \leq L_{k_{c}}\left|u_{1}-u_{2}\right|$, for all $u_{1}, u_{2} \in \mathbb{R}$.

(H6) The normal compliance function $p_{\nu}$ and the friction bound $p_{\tau}$ satisfy the following hypothesis for $r=\nu, \tau$

i) $p_{r}: \Gamma_{C} \times \mathbb{R} \rightarrow \mathbb{R}_{+}$,

ii) $x \rightarrow p_{r}(x, u)$ is measurable on $\Gamma_{C}$, for all $u \in \mathbb{R}$,

iii) $x \rightarrow p_{r}(x, u)=0$ for $u \leq 0$, a.e. $x \in \Gamma_{C}$,

iv) There exists $L_{r}>0$ such that $\left|p_{r}(., u)-p_{r}(., v)\right| \leq L_{r}|u-v|$, for all $u, v \in \mathbb{R}_{+}$.

i) The initial data $u_{0}$ and $\dot{u}_{0}$ of Problem $(P)$ satisfy

$$
u_{0} \in V_{a d}, \quad \dot{u}_{0} \in D(\partial j),
$$

where $\partial j$ denotes the subdifferential of $j$ and $D(\partial j)$ represents its domain.

ii) There exists $h \in L^{2}(\Omega)^{d}$ such that

$$
b\left(\dot{u}_{0}, v-\dot{u}_{0}\right)+a\left(u_{0}, v-\dot{u}_{0}\right)+j\left(u_{0}, v\right)-j\left(u_{0}, \dot{u}_{0}\right) \geq\left(h, v-\dot{u}_{0}\right), \forall v \in V .
$$

According to this notation and by using a standard procedure based on Green's formula, we can state the variational formulation of (2.1)-(2.11), in terms of displacement field and temperature.

Problem $(P V)$ : Find a displacement field $u: \Omega \times] 0, T\left[\longrightarrow \mathbb{R}^{d}\right.$ and a temperature $\theta: \Omega \times] 0, T[\longrightarrow \mathbb{R}$ a.e. $t \in] 0, T[, v \in V$ and $\eta \in Q$ such that

$$
\begin{gathered}
(\ddot{u}(t), v-\dot{u}(t))+a(u(t), v-\dot{u}(t))+b(\dot{u}(t), v-\dot{u}(t))-m(\theta(t), v-\dot{u}(t)) \\
+j(u(t), v)-j(u(t), \dot{u}(t)) \geq(f(t), v-\dot{u}(t))_{V}, \\
(\dot{\theta}(t), \eta)+d(\theta(t), \eta)-e(u(t), \eta)+\chi(u(t), \theta(t), \eta)=\left(q_{t h}(t), \eta\right)_{Q}, \\
u(0)=u_{0}, \dot{u}(0)=\dot{u}_{0}, \theta(0)=\theta_{0} .
\end{gathered}
$$

\section{An existence And Uniqueness Result}

In this section, we present and we prove an existence and uniqueness result.

Theorem 4.1. Assume that the assumptions (H1)-(H7) hold. Then, there exists a unique solution $(u, \theta)$ to problem (3.19)-(3.21) that satisfies

$$
\begin{array}{r}
u \in W^{1, \infty}(0, T ; V) \cap W^{2, \infty}(0, T ; H), \\
\quad \theta \in L^{2}(0, T ; Q) \cap C\left([0, T] ; L^{2}(\Omega)\right) .
\end{array}
$$

The proof of this theorem is carried out in several steps and it is based on argument of second-order non linear evolution variational inequality and Banach fixed point theorem. Let $\alpha \in L^{2}(0, T ; V)$ and $\beta \in L^{2}\left(0, T ; L^{2}(\Omega)\right)$ given by

$$
\begin{array}{r}
\left(\alpha(t), v-\dot{u}_{\alpha}(t)\right)=m\left(\theta_{\beta}(t), v-\dot{u}_{\alpha}(t)\right), \\
(\beta(t), \eta)=-e\left(u_{\alpha}(t), \eta\right)+\chi\left(u_{\alpha}(t), \theta_{\beta}(t), \eta\right) .
\end{array}
$$

In the first, we consider the following variational problem of displacement field

Problem $\left(P V_{d f}\right)$ : Find a displacement field $\left.u_{\alpha}: \Omega \times\right] 0, T\left[\longrightarrow \mathbb{R}^{d}\right.$ a.e. $\left.t \in\right] 0, T[$ such that

$$
\begin{gathered}
\left(\ddot{u}_{\alpha}(t), v-\dot{u}_{\alpha}(t)\right)+a\left(u_{\alpha}(t), v-\dot{u}_{\alpha}(t)\right)+b\left(\dot{u}_{\alpha}(t), v-\dot{u}_{\alpha}(t)\right), \\
-\left(\alpha(t), v-\dot{u}_{\alpha}(t)\right)+j\left(u_{\alpha}(t), v\right)-j\left(u_{\alpha}(t), \dot{u}_{\alpha}(t)\right) \geq\left(f(t), v-\dot{u}_{\alpha}(t)\right)_{V}, \forall v \in V, \\
u_{\alpha}(0)=u_{0}, \dot{u}_{\alpha}(0)=\dot{u}_{0} .
\end{gathered}
$$

We have the following existence and uniqueness result. 
Lemma 4.2. For all $v \in V_{a d}$ and for a.e $\left.t \in\right] 0, T[$, the problem (4.26) -(4.27) has a unique solution $u_{\alpha}$ satisfies the regularity (4.22).

Proof. By Riesz's representation theorem, we define the operator

$$
\left(f_{\alpha}(t), v\right)_{V}=(f(t), v)_{V}-(\alpha(t), v)_{V} .
$$

Then (4.26)- (4.27) can be written

$$
\begin{gathered}
\left(\ddot{u}_{\alpha}(t), v-\dot{u}_{\alpha}(t)\right)+b\left(\dot{u}_{\alpha}(t), v-\dot{u}_{\alpha}(t)\right)+a\left(u_{\alpha}(t), v-\dot{u}_{\alpha}(t)\right) \\
\quad+j\left(u_{\alpha}(t), v\right)-j\left(u_{\alpha}(t), \dot{u}_{\alpha}(t)\right) \geq\left(f(t), v-\dot{u}_{\alpha}(t)\right)_{V} .
\end{gathered}
$$

By assumptions (H1) and (H2) the operator $a$ is continuous symmetric bilinear and coercive.

Next, we define the set-valued operator $B: V \mapsto V$ by $B=b+\partial j$.

From (H2), $b$ is maximal monotone and by (H4)(iv), we observe that $\partial j$ is maximal monotone. Consequently, [5. P. 39] the operator $B$ is maximal monotone.

Moreover, by (H7), the initial data $u_{0}$ and $\dot{u}_{0}$ satisfy the condition $\left\{a u_{0}+b \dot{u}_{0}\right\} \cap L^{2}(\Omega)^{d} \neq$ $\emptyset$.

Keeping in mind the regularity $f \in W^{1,1}(0, T ; H)$ and $\alpha \in L^{2}(0, T ; V)$, we deduce from (4.28) that $f_{\alpha} \in W^{1,1}(0, T ; H)$.

Finally, using the Theorem presented in [5, P. 268], we obtain result.

In the second step, we consider the following variational problem of the temperature. Problem $\left(P V_{t h}\right):$ Find a temperature $\left.\theta_{\beta}: \Omega \times\right] 0, T[\longrightarrow \mathbb{R}$ a.e. $t \in] 0, T[$ and $\eta \in Q$ such that

$$
\begin{gathered}
\left(\dot{\theta}_{\beta}(t), \eta\right)+d\left(\theta_{\beta}(t), \eta\right)+(\beta(t), \eta)=\left(q_{t h}(t), \eta\right)_{Q}, \\
\theta_{\beta}(0)=\theta_{0} .
\end{gathered}
$$

The result of this problem presented in the following lemma.

Lemma 4.3. There exists a unique solution for the problem (4.30)-(4.31) which satisfies (4.23).

Proof. Using Riesz's representation theorem, there exists an operator $q_{\beta}$ defined by

$$
\left(q_{\beta}(t), \eta\right)_{Q}=\left(q_{t h}(t), \eta\right)_{Q}-(\beta(t), \eta)_{Q} .
$$

Then, the problem (4.30)-(4.31) can be written as follows

$$
\begin{gathered}
\left(\dot{\theta}_{\beta}(t), \eta\right)+d\left(\theta_{\beta}(t), \eta\right)=\left(q_{\beta}(t), \eta\right), \\
\theta_{\beta}(0)=\theta_{0} .
\end{gathered}
$$

From (H1)-(H2) the operator $d$ is a hemicontinuous and monotone. By (4.32) and the regularity of $q_{t h}$, we obtain that $q_{\beta} \in L^{2}(0, T ; Q)$.

It follows now from Theorem presented [20, P. 48] that there exists a unique function $\theta_{\beta}$ which satisfies (4.23).

In the last step, for all $t \in[0, T]$ we define the operator

$$
\Lambda(\alpha, \beta)(t):=\left(\Lambda_{1}(\alpha, \beta)(t), \Lambda_{2}(\alpha, \beta)(t)\right) \in V \times Q,
$$

given by

$$
\begin{aligned}
& \left(\Lambda_{1}(\alpha, \beta)(t), v\right):=m\left(\theta_{\beta}, v\right), \\
& \left(\Lambda_{2}(\alpha, \beta)(t), \eta\right):=-e\left(u_{\alpha}(t), \eta\right)+\chi\left(u_{\alpha}(t), \theta_{\beta}(t), \eta\right),
\end{aligned}
$$

and we have the following result

Lemma 4.4. For $(\alpha, \beta) \in L^{2}(0, T ; V) \times L^{2}(0, T ; Q)$ the operator $\Lambda$ is continuous. Moreover, there exists a unique $\left(\alpha^{*}, \beta^{*}\right) \in L^{2}(0, T ; V \times Q)$ such that $\Lambda\left(\alpha^{*}, \beta^{*}\right)=\left(\alpha^{*}, \beta^{*}\right)$. 
Proof. Let $(\alpha, \beta) \in L^{2}(0, T ; V \times Q)$ and let $t_{1}, t_{2} \in[0, T]$.

From (4.36) and assumption (H3), it follows that

$$
\left\|\Lambda_{1}(\alpha, \beta)\left(t_{1}\right)-\Lambda_{1}(\alpha, \beta)\left(t_{2}\right)\right\|_{V \times Q} \leq M_{m}\left\|\theta_{\beta}\left(t_{1}\right)-\theta_{\beta}\left(t_{2}\right)\right\|_{Q} .
$$

Taking into account the regularity of $\theta_{\beta}$, we found that $\Lambda_{1}(\alpha, \beta) \in C([0, T] ; V)$.

The relation (4.37) combined with ( $\mathrm{H} 3),(\mathrm{H} 5)$ and (3.14), there exists a positive constant $c$ depending on $M_{e}, M_{m}, L, M_{k c}, C_{S_{1}}$ and $C_{S_{2}}$ such that

$$
\begin{aligned}
& \left\|\Lambda_{2}(\alpha, \beta)\left(t_{1}\right)-\Lambda_{2}(\alpha, \beta)\left(t_{2}\right)\right\|_{V \times Q} \\
& \leq c\left(\left\|u_{\alpha}\left(t_{1}\right)-u_{\alpha}\left(t_{2}\right)\right\|_{V}+\left\|\theta_{\beta}\left(t_{1}\right)-\theta_{\beta}\left(t_{2}\right)\right\|_{Q}\right) .
\end{aligned}
$$

Then, $\Lambda_{2}(\alpha, \beta) \in C([0, T], Q)$. Consequently, we deduce that $\Lambda$ is continuous.

Let $\left(\alpha_{1}, \beta_{1}\right),\left(\alpha_{2}, \beta_{2}\right) \in L^{2}(0, T ; V \times Q)$ and $t \in[0, T]$. Similar to (4.38) and (4.39), we have that

$$
\begin{aligned}
& \left\|\Lambda\left(\alpha_{1}, \beta_{1}\right)(t)-\Lambda\left(\alpha_{2}, \beta_{2}\right)(t)\right\|_{V \times Q}^{2} \\
& \leq c\left(\left\|u_{\alpha_{1}}(t)-u_{\alpha_{2}}(t)\right\|_{V}^{2}+\left\|\theta_{\beta_{1}}(t)-\theta_{\beta_{2}}(t)\right\|_{Q}^{2}\right) .
\end{aligned}
$$

Also from $u_{\alpha_{i}}(t)=\int_{0}^{t} \dot{u}_{\alpha_{i}}(s) d s+u_{0}$ for $i=1,2$, we found

$$
\left\|u_{\alpha_{1}}(t)-u_{\alpha_{2}}(t)\right\|_{V}^{2} \leq c \int_{0}^{t}\left\|\dot{u}_{\alpha_{1}}(s)-u_{\alpha_{2}}(s)\right\|_{V}^{2} d s .
$$

Using inequality (4.26), we find

$$
\begin{gathered}
\left(\ddot{u}_{\alpha_{1}}(t)-\ddot{u}_{\alpha_{2}}(t), \dot{u}_{\alpha_{1}}(t)-\dot{u}_{\alpha_{2}}(t)\right)+b\left(\dot{u}_{\alpha_{1}}(t)-\dot{u}_{\alpha_{2}}(t), \dot{u}_{\alpha_{1}}(t)-\dot{u}_{\alpha_{2}}(t)\right) \\
a\left(u_{\alpha_{1}}(t)-u_{\alpha_{2}}(t), \dot{u}_{\alpha_{1}}(t)-\dot{u}_{\alpha_{2}}(t)\right)+\left(\alpha_{1}(t)-\alpha_{2}(t), \dot{u}_{\alpha_{1}}(t)-\dot{u}_{\alpha_{2}}(t)\right) \\
j\left(u_{\alpha_{1}}(t), \dot{u}_{\alpha_{1}}(t)\right)-j\left(u_{\alpha_{1}}(t), \dot{u}_{\alpha_{2}}(t)\right)-j\left(u_{\alpha_{2}}(t), \dot{u}_{\alpha_{1}}(t)\right)+j\left(u_{\alpha_{2}}(t), \dot{u}_{\alpha_{2}}(t)\right) \leq 0 .
\end{gathered}
$$

Moreover, from (3.14), (3.17) and (H5), we have

$$
\begin{gathered}
\left|j\left(u_{\alpha_{1}}(t), \dot{u}_{\alpha_{1}}(t)\right)-j\left(u_{\alpha_{1}}(t), \dot{u}_{\alpha_{2}}(t)\right)-j\left(u_{\alpha_{2}}(t), \dot{u}_{\alpha_{1}}(t)\right)+j\left(u_{\alpha_{2}}(t), \dot{u}_{\alpha_{2}}(t)\right)\right| \\
\leq C_{S_{1}}^{2}\left(L_{\nu}+L_{\tau}\right)\left\|u_{\alpha_{1}}(t)-u_{\alpha_{2}}(t)\right\|_{V}\left\|\dot{u}_{\alpha_{1}}(t)-\dot{u}_{\alpha_{2}}(t)\right\|_{V} .
\end{gathered}
$$

We integrate the relation (4.42) to 0 at $t$ and use (4.43), (H2) and the initial condition $\dot{u}_{\alpha_{1}}(0)=\dot{u}_{\alpha_{2}}(0)=\dot{u}_{0}$ to obtain

$$
\begin{aligned}
& m_{b} \int_{0}^{t}\left\|\dot{u}_{\alpha_{1}}(s)-\dot{u}_{\alpha_{2}}(s)\right\|_{V}^{2} d s+\frac{1}{2}\left\|\dot{u}_{\alpha_{1}}(t)-\dot{u}_{\alpha_{2}}(t)\right\|_{V}^{2} \\
& \leq-\int_{0}^{t}\left(\alpha_{1}(s)-\alpha_{2}(s), \dot{u}_{\alpha_{1}}(t)-\dot{u}_{\alpha_{2}}(t)\right) d s \\
& +\left(M_{a}+C_{S_{1}}^{2}\left(L_{\nu}+L_{\tau}\right)\right) \int_{0}^{t}\left\|u_{\alpha_{1}}(s)-u_{\alpha_{2}}(s)\right\|_{V} \cdot\left\|\dot{u}_{\alpha_{1}}(s)-\dot{u}_{\alpha_{2}}(s)\right\|_{V} d s .
\end{aligned}
$$

Then, using the inequality $x y \leq \kappa x^{2}+\frac{1}{4 \kappa} y^{2},(\kappa>0)$ and Gornwall inequality, we get that

$$
\left\|u_{\alpha_{1}}(t)-u_{\alpha_{2}}(t)\right\|_{V}^{2} \leq c \int_{0}^{t}\left\|\alpha_{1}(s)-\alpha_{2}(s)\right\|_{V}^{2} d s .
$$

In order words from (4.26), it follows that

$$
\begin{gathered}
\left(\dot{\theta}_{\beta_{1}}(t)-\dot{\theta}_{\beta_{2}}(t), \theta_{\beta_{1}}(t)-\theta_{\beta_{2}}(t)\right)+d\left(\theta_{\beta_{1}}(t)-\theta_{\beta_{2}}(t), \theta_{\beta_{1}}(t)-\theta_{\beta_{2}}(t)\right) \\
+\left(\beta_{1}(t)-\beta_{2}(t), \theta_{\beta_{1}}(t)-\theta_{\beta_{2}}(t)\right)=0 .
\end{gathered}
$$


Similar to (4.45), we have

$$
\left\|\theta_{\beta_{1}}(t)-\theta_{\beta_{2}}(t)\right\|_{Q}^{2} \leq c \int_{0}^{t}\left\|\beta_{1}(s)-\beta_{2}(s)\right\|_{Q}^{2} d s .
$$

Now, we combine (4.40), (4.45) and (4.47), we obtain

$$
\left\|\Lambda\left(\alpha_{1}, \beta_{1}\right)(t)-\Lambda\left(\alpha_{2}, \beta_{2}\right)(t)\right\|_{V \times Q}^{2} \leq c \int_{0}^{t}\left\|\left(\alpha_{1}, \beta_{1}\right)(s)-\left(\alpha_{2}, \beta_{2}\right)(s)\right\|_{V \times Q}^{2} d s .
$$

Reiterating this inequality $n$ times result in

$$
\begin{aligned}
& \left\|\Lambda^{n}\left(\alpha_{1}, \beta_{1}\right)-\Lambda^{n}\left(\alpha_{2}, \beta_{2}\right)\right\|_{L^{2}(0, T ; V \times Q)}^{2} \\
& \leq \frac{(C T)^{n}}{n !}\left\|\left(\alpha_{1}, \beta_{1}\right)-\left(\alpha_{2}, \beta_{2}\right)\right\|_{L^{2}(0, T ; V \times Q)}^{2} .
\end{aligned}
$$

Which implies that for $n$ sufficiently large, $\Lambda^{n}$ is a contraction operator in the Banach space $L^{2}(0, T ; V \times Q)$.

Therefore, $\Lambda$ has a unique fixed point.

Now we have everything that is required to prove Theorem (4.1)

Proof of Theorem (4.1).

Existence: Let $\left(\alpha^{*}, \beta^{*}\right) \in L^{2}\left(0, T ; V \times L^{2}(\Omega)\right)$ be the fixed point of the operator $\Gamma$ and denote $u_{\alpha^{*}}^{*}, \theta_{\beta^{*}}^{*}$ be the solution of (4.26)-(4.27) and (4.30)-(4.31) respectively, for $(\alpha, \beta)=\left(\alpha^{*}, \beta^{*}\right)$, the definition of $\Lambda$ we find that the pair $\left(u_{\alpha^{*}}^{*}, \theta_{\beta^{*}}^{*}\right)$ is a solution of Problem $(P V)$.

Uniqueness: The uniqueness of the solution is a consequence of the fixed point of operator $\Lambda$ given by (4.35).

\section{Continuous dependence of Problem disturbed}

In this section we consider $p_{\nu}^{\delta}$ and $p_{\tau}^{\delta}$ for any $\delta>0$ the perturbation of $p_{\nu}$ and $p_{\tau}$ which satisfies (H6) and we study the dependence of the solution to (3.19)-(3.21).

We define the functional $j^{\delta}$ from $j$ by replacing $p_{\nu}$ and $p_{\tau}$ with $p_{\nu}^{\delta}$ and $p_{\tau}^{\delta}$. We introduce the following variational problem

Problem $\left(P V^{\delta}\right)$ : For $\delta>0$ find a displacement field $\left.u^{\delta}: \Omega \times\right] 0, T\left[\longrightarrow \mathbb{R}^{d}\right.$ and a temperature $\left.\theta^{\delta}: \Omega \times\right] 0, T[\longrightarrow \mathbb{R}$ a.e. $t \in] 0, T[, v \in V$ and $\eta \in Q$ such that

$$
\begin{gathered}
\left(\ddot{u}^{\delta}(t), v-\dot{u}^{\delta}(t)\right)+a\left(u^{\delta}(t), v-\dot{u}^{\delta}(t)\right)+b\left(\dot{u}^{\delta}(t), v-\dot{u}^{\delta}(t)\right) \\
-m\left(\theta^{\delta}(t), v-\dot{u}^{\delta}(t)\right)+j^{\delta}\left(u^{\delta}(t), v\right)-j^{\delta}\left(u^{\delta}(t), \dot{u}^{\delta}(t)\right) \geq\left(f(t), v-\dot{u}^{\delta}(t)\right)_{V}, \\
\left(\dot{\theta}^{\delta}(t), \eta\right)+d\left(\theta^{\delta}(t), \eta\right)-e\left(u^{\delta}(t), \eta\right)+\chi\left(u^{\delta}(t), \theta^{\delta}(t), \eta\right)=\left(q_{t h}(t), \eta\right)_{Q}, \\
u^{\delta}(0)=u_{0}, \dot{u}^{\delta}(0)=\dot{u}_{0}, \theta^{\delta}(0)=\theta_{0} .
\end{gathered}
$$

This problem has a unique solution, and the proof is similar to that used in Section 4. We present now, the assumptions of the contact function $p_{\nu}^{\delta}$ and $p_{\tau}^{\delta}$

$$
\left\{\begin{array}{l}
\text { There exists } \omega_{\tau} \in \mathbb{R} \text { and } \varphi_{\tau}: \mathbb{R}_{+} \rightarrow \mathbb{R}_{+} \text {such that: } \\
(i)\left|p_{\tau}^{\delta}(x, r)-p_{\tau}(x, r)\right| \leq \varphi_{\tau}(\delta)\left(|r|+\omega_{\tau}\right), \quad \forall r \in \mathbb{R}^{N}, \text { a.e. } x \in \Gamma_{C}, \\
\left(\text { ii) } \lim _{\delta \rightarrow 0} \varphi_{\tau}(\delta)=0\right.
\end{array}\right.
$$

and

$$
\left\{\begin{array}{l}
\text { There exists } \omega_{\nu} \in \mathbb{R} \text { and } \varphi_{\nu}: \mathbb{R}_{+} \rightarrow \mathbb{R}_{+} \text {such that: } \\
(i)\left|p_{\nu}^{\delta}(x, r)-p_{\nu}(x, r)\right| \leq \varphi_{\nu}(\delta)\left(|r|+\omega_{\nu}\right), \quad \forall r \in \mathbb{R}^{N}, \text { a.e. } x \in \Gamma_{C}, \\
(\text { ii }) \lim _{\delta \rightarrow 0} \varphi_{\nu}(\delta)=0
\end{array}\right.
$$

Next, we present the following convergence result 
Theorem 5.1. The solution $\left(u^{\delta}, \theta^{\delta}\right)$ of (5.50)-(5.52) converge uniformly to a solution of (3.19) - (3.21) i.e.,

$$
u^{\delta} \longrightarrow u, \dot{u}^{\delta} \longrightarrow \dot{u}, \text { and } \theta^{\delta} \longrightarrow \theta \text { as } \delta \longrightarrow 0 .
$$

Proof. Replacing $v$ by $\dot{u}^{\delta}$ in (3.19) and by $\dot{u}$ in (5.50), we have

$$
\begin{gathered}
\left(\ddot{u}(t)-\ddot{u}^{\delta}(t), \dot{u}(t)-\dot{u}^{\delta}(t)\right)+a\left(u(t)-u^{\delta}(t), \dot{u}(t)-\dot{u}^{\delta}(t)\right) \\
+b\left(\dot{u}(t)-\dot{u}^{\delta}(t), \dot{u}(t)-\dot{u}^{\delta}(t)\right)-m\left(\theta(t)-\theta^{\delta}(t)\right) \\
j(u(t), \dot{u}(t))-j\left(u(t), \dot{u}^{\delta}(t)\right)+j^{\delta}\left(u^{\delta}(t), \dot{u}^{\delta}(t)\right)-j^{\delta}\left(u^{\delta}(t), \dot{u}(t)\right) \leq 0 .
\end{gathered}
$$

Using (H2) and (H3) we found the inequality

$$
\begin{aligned}
& m_{b}\left\|\dot{u}(t)-\dot{u}^{\delta}(t)\right\|_{V}^{2}+\frac{1}{2} \frac{d}{d t}\left\|\dot{u}(t)-\dot{u}^{\delta}(t)\right\|_{V}^{2} \\
& \leq\left(M_{a}+M_{m}\right)\left\|\dot{u}(t)-\dot{u}^{\delta}(t)\right\|_{V}\left(\left\|u(t)-u^{\delta}(t)\right\|_{V}+\left\|\theta(t)-\theta^{\delta}(t)\right\|_{Q}\right)+\left|R_{j, j^{\delta}}\right| .
\end{aligned}
$$

The assumption (5.53)-(5.54) and (3.17) allows us to obtain

$$
\begin{aligned}
\left|R_{j, j^{\delta}}\right| & =\mid \int_{\Gamma_{C}}\left(p_{\nu}\left(u_{\nu}(t)-g\right)-p_{\nu}^{\delta}\left(u_{\nu}^{\delta}(t)-g\right)\right)\left(\dot{u}_{\nu}^{\delta}(t)-\dot{u}_{\nu}(t)\right) d a \\
& +\int_{\Gamma_{C}}\left(p_{\nu}\left(u_{\nu}(t)-g\right)-p_{\nu}^{\delta}\left(u_{\nu}^{\delta}(t)-g\right)\right)\left(\left\|\dot{u}_{\nu}^{\delta}(t)\right\|-\left\|\dot{u}_{\nu}(t)\right\|\right) d a \mid \\
& \leq c\left(\varphi_{\nu}(\delta)+\varphi_{\tau}(\delta)\right)\left\|\dot{u}^{\delta}(t)-\dot{u}(t)\right\|_{V} .
\end{aligned}
$$

Combining (5.56), (5.57) and using Gronwall inequality, it follows that

$$
\begin{aligned}
& \left\|u(t)-u^{\delta}(t)\right\|_{V}^{2}+\left\|\dot{u}(t)-\dot{u}^{\delta}(t)\right\|_{V}^{2} \\
& \leq c\left(\int_{0}^{t}\left\|\theta(s)-\theta^{\delta}(s)\right\|_{Q}^{2} d s+\left(\varphi_{\nu}(\delta)+\varphi_{\tau}(\delta)\right)\right) .
\end{aligned}
$$

Now, we replace $\eta$ by $\theta-\theta^{\delta}$ in (3.20) and in (5.51), we have the following relation

$$
\begin{aligned}
& \left(\dot{\theta}(t)-\dot{\theta}^{\delta}(t), \theta(t)-\theta^{\delta}(t)\right)+d\left(\theta(t)-\theta^{\delta}(t), \theta(t)-\theta^{\delta}(t)\right) \\
& -e\left(u(t)-u^{\delta}(t), \theta(t)-\theta^{\delta}(t)\right)+\chi\left(u(t), \theta(t), \theta(t)-\theta^{\delta}(t)\right) \\
& -\chi\left(u^{\delta}(t), \theta^{\delta}(t), \theta(t)-\theta^{\delta}(t)\right)=0 .
\end{aligned}
$$

Using the property of operator $d$ and $e$, we deduce that

$$
\begin{aligned}
& m_{d}\left\|\theta(t)-\theta^{\delta}(t)\right\|_{Q}^{2}+\frac{1}{2} \frac{d}{d t}\left\|\theta(t)-\theta^{\delta}(t)\right\|_{Q}^{2} \leq \\
& M_{e}\left\|u(t)-u^{\delta}(t)\right\|_{V}\left\|\theta(t)-\theta^{\delta}(t)\right\|_{Q}+\left|R_{\chi}\right| .
\end{aligned}
$$

From (H4) and (3.14), we have the following estimate of $R_{\chi}$

$$
\left|R_{\chi}\right| \leq M_{k_{c}} \cdot L \cdot C_{S_{2}}^{2}\left\|\theta(t)-\theta^{\delta}(t)\right\|_{Q}^{2} .
$$

Similar to (5.59), we conclude that

$$
\left\|\theta(t)-\theta^{\delta}(t)\right\|_{Q}^{2} \leq c \int_{0}^{t}\left\|u(s)-u^{\delta}(s)\right\|_{V}^{2} d s
$$

Moreover, from (5.59) and (5.62) combined with Gronwall inequality we find

$$
\left\|u(t)-u^{\delta}(t)\right\|_{V}^{2}+\left\|\dot{u}(t)-\dot{u}^{\delta}(t)\right\|_{V}^{2}+\left\|\theta(t)-\theta^{\delta}(t)\right\|_{Q}^{2} \leq c\left(\varphi_{\nu}(\delta)+\varphi_{\tau}(\delta)\right) .
$$

Hence, since $\delta \longrightarrow 0$, we obtain the result of Theorem (5.1). 


\section{REFERENCES}

[1] Samir Adly, Oanh Chau, and Mohamed Rochdi, Solvability of a class of thermal dynamical contact problems with subdifferential conditions, (2012).

[2] A Amassad, KL Kuttler, M Rochdi, and M Shillor, Quasi-static thermoviscoelastic contact problem with slip dependent friction coefficient, Mathematical and computer modelling 36 (2002), no. 7-8, 839-854.

[3] KT Andrews, KL Kuttler, M Rochdi, and M Shillor, One-dimensional dynamic thermoviscoelastic contact with damage, Journal of mathematical analysis and applications 272 (2002), no. 1, 249-275.

[4] B Awbi, El H Essoufi, and M Sofonea, A viscoelastic contact problem with normal damped response and friction, Annales Polonici Mathematici, vol. 75, Instytut Matematyczny Polskiej Akademii Nauk, 2000, pp. 233-246.

[5] V. Barbu, Nonlinear semigroups and differential equations in banach spacese, (1976).

[6] Oanh Chau and Viorica Venera Motreanu, Dynamic contact problems with velocity conditions, International Journal of applied mathematics and computer science 12 (2002), 17-26.

[7] Oanh Chau, Rachid Oujja, and Mohamed Rochdi, A mathematical analysis of a dynamical frictional contact model in thermoviscoelasticity, Discrete \& Continuous Dynamical Systems-S 1 (2008), no. 1, 61.

[8] G Duvaut, Free boundary problem connected with thermoelasticity and unilateral contact, Free boundary problems 2 (1979), 217-236.

[9] IR Ionescu and J-C Paumier, On the contact problem with slip rate dependent friction in elastodynamics, European journal of mechanics. A. Solids 13 (1994), no. 4, 555-568.

[10] Jiří Jarušek, Dynamic contact problems with given friction for viscoelastic bodies, Czechoslovak Mathematical Journal 46 (1996), no. 3, 475-487.

[11] Mostafa Kabbaj and El-H Essoufi, Frictional contact problem in dynamic electroelasticity, Glasnik matematički 43 (2008), no. 1, 137-158.

[12] Mostafa Kabbaj and El-Hassan Essoufi, Slip-dependent friction in dynamic electroviscoelasticity, Glasnik matematički 45 (2010), no. 1, 125-137.

[13] KL Kuttler and Meir Shillor, A dynamic contact problem in one-dimensional thermoviscoelasticity, Nonlinear World 2 (1995), 355-385.

[14] Stanislaw Migorski, Boundary hemivariational inequalities of hyperbolic type and applications, Journal of Global Optimization 31 (2005), no. 3, 505-533.

[15] Stanisław Migórski*, Dynamic hemivariational inequality modeling viscoelastic contact problem with normal damped response and friction, Applicable Analysis 84 (2005), no. 7, 669-699.

[16] Stanisław Migórski, Anna Ochal, and Mircea Sofonea, Integrodifferential hemivariational inequalities with applications to viscoelastic frictional contact, Mathematical Models and Methods in Applied Sciences 18 (2008), no. 02, 271-290.

[17] Stanisław Migórski and Paweł Szafraniec, A class of dynamic frictional contact problems governed by a system of hemivariational inequalities in thermoviscoelasticity, Nonlinear Analysis: Real World Applications 15 (2014), 158-171.

[18] Roberto Giorgio Rabel, Weimin Han, and Mircea Sofonea, Quasistatic contact problems in viscoelasticity and viscoplasticity, American Mathematical Soc., 2002.

[19] M Rochdi and M Shillor, Existence and uniqueness for a quasistatic frictional bilateral contact problem in thermoviscoelasticity, Quarterly of Applied Mathematics 58 (2000), no. 3, 543-560.

[20] Mircea Sofonea, Weimin Han, and Meir Shillor, Analysis and approximation of contact problems with adhesion or damage, CRC Press, 2005.

[21] Paweł Szafraniec, Dynamic nonsmooth frictional contact problems with damage in thermoviscoelasticity, Mathematics and Mechanics of Solids 21 (2016), no. 5, 525-538.

Mustapha Bouallala: bouallalamustaphaan@gmail.com

Department of Mathematics and Computer Science, Polydisciplinary faculty of Safi, Cadi Ayyad University, Marrakech, Morocco

El Hassan Essoufi: e.h.essoufi@gmail.com

Univ. Hassan 1, Laboratory MISI, 26000 Settat, Morocco.

Received 18/06/2020; Revised 21/10/2020 